\title{
Supplementary Information for Shift of the Optimum Binding Energy at Higher Rates of Catalysis
}

\author{
Hideshi Ooka ${ }^{1 *}$, Ryuhei Nakamura1,2 \\ *hideshi.ooka@riken.jp
}

\author{
${ }^{1}$ Biofunctional Catalyst Research Team, Center for Sustainable Resource Science (CSRS), \\ RIKEN \\ 2 - 1, Hirosawa, Wako, Saitama 351 - 0198, Japan \\ Phone: +81-(0)48-467-9372 \\ Fax: +81-(0)48-462-4639 \\ 2 Earth-Life Science Institute (ELSI), Tokyo Institute of Technology, \\ 2 - 12 - IE - 1, Ookayama, Meguro-ku, Tokyo 152 - 8550, Japan
}

This file includes:

Appendix A: Validity of the Steady-State Approximation

Appendix B: Definition of "Large" Driving Force

Appendix C: Alternative Method of Obtaining the Optimum Binding Energy

Appendix D: Value of Optimum $K$ for a Given Binding Energy 


\section{Appendix A: Validity of the Steady-State Approximation}

As stated in the main text, there are currently two major approaches to calculate the kinetics of chemical reactions: the pre-equilibrium assumption and the steady-state approximation. Here, we have used the steady-state approximation because we also intended to consider reaction conditions where a pre-equilibrium does not exist (i.e., a mechanism where the rate-limiting step and the step preceding it have similar kinetics).

The main argument against the steady-state approximation is that it implicitly assumes that the intermediate concentration is infinitely small relative to the concentration of the reactant and product. ${ }^{1}$ This is true when there is a closed system with a fixed amount of reactant.

Namely, in a closed system, mass balance indicates

$$
2\left[\mathrm{H}_{2}\right]+\left[\mathrm{H}_{\mathrm{ads}}\right]+\left[\mathrm{H}^{+}\right]=\text {const. }
$$

The initial concentration of the intermediate $\left[\mathrm{H}_{\mathrm{ads}}\right]$ and product $\left[\mathrm{H}^{+}\right]$can be assumed to be zero before the reaction occurs. As the steady state approximation sets $\frac{\partial\left[\mathrm{H}_{\mathrm{ads}}\right]}{\partial t}$ to zero, this indicates that $2 \frac{\partial\left[\mathrm{H}_{2}\right]}{\partial t}+\frac{\partial\left[\mathrm{H}^{+}\right]}{\partial t}=0$. Integration leads to $\left[\mathrm{H}_{2}\right]+\left[\mathrm{H}^{+}\right]=\left[\mathrm{H}_{2}\right]_{\mathrm{t}=0}$. This is compatible with Eq. S1 only if $\left[\mathrm{H}_{2}\right]>>\left[\mathrm{H}_{\text {ads }}\right]$.

However, in the case of an open system, the boundary conditions indicated by Eq. S1 are not necessarily required, and under these conditions, the coverage of the intermediate can increase without conflict. 


\section{Appendix B: Definition of "Large" Driving Force}

The condition of large $E$ is equivalent to the case where the back reaction of reaction 1 can be ignored $\left(k_{2}>>k_{-1}\right)$ as shown below:

$$
\begin{gathered}
\frac{\exp (2 F E)}{K}=\frac{k_{2}^{0} \exp (f E)}{k_{1}^{0} \exp (-f E)} * \frac{\exp \left(f E_{1}\right)}{\exp \left(f E_{1}\right)}=\frac{\mathrm{k}_{2}}{k_{-1}} \\
\therefore \exp (2 f E) \gg K \leftrightarrow k_{2} \gg k_{-1}
\end{gathered}
$$

Eq. S3

In this case, the back reaction of reaction 2 can also be ignored $\left(k_{2}>>k_{-2}\right)$, because:

$$
\frac{k_{2}}{k_{-2}}=\frac{\exp \left(f\left(E+E_{1}\right)\right)}{\exp \left(f\left(-E-E_{1}\right)\right)}=\exp \left(2 f\left(E+E_{1}\right)\right) \gg 1
$$

Under this condition, the two forward reactions must be equal rate to yield a steady state, as can be confirmed easily below:

$$
\begin{array}{cc}
v_{1}=k_{1}^{0} \exp \left(f E-f E_{1}\right)=k_{1}^{0} \exp (f E) \frac{1}{\sqrt{K}}=\sqrt{k_{1}^{0} k_{2}^{0}} \exp (f E) & \text { Eq. S5 } \\
v_{2}=k_{2}^{0} \exp \left(f E+f E_{1}\right)=k_{2}^{0}(f E) \sqrt{K}=\sqrt{k_{1}^{0} k_{2}^{0}} \exp (f E) & \text { Eq. S6 }
\end{array}
$$




\section{Appendix C: Alternative Method of Obtaining the Optimum Binding Energy}

Although the most direct and intuitive method of obtaining the maximum current in Eq. 20 of the main text is to take the derivative of the denominator as shown in the main text, a more mathematically facile approach is shown below.

As explained in the main text, the goal is to obtain the conditions for $A$ and $B$ which allow the denominator to be minimized.

$$
(\text { denominator })=\left(K A+\frac{1}{A}\right) \frac{1}{B}+\left(\frac{K}{A}+A\right) B
$$

For two positive values $x$ and $y$, their arithmetic mean is larger than their geometric mean, with the equality being satisfied when $x=y$. By taking

$$
x=\left(K A+\frac{1}{A}\right) \frac{1}{B}
$$

and

$$
y=\left(\frac{K}{A}+A\right) B
$$

we obtain

$$
\text { (denominator }) \geq \sqrt{\left(K A+\frac{1}{A}\right)\left(\frac{K}{A}+A\right)}
$$

with the equality being satisfied when

$$
B=\sqrt{\frac{K A+\frac{1}{A}}{\frac{K}{A}+A}}=\sqrt{\frac{K A^{2}+1}{K+A^{2}}}
$$

This is equivalent to the optimum binding energy obtained in the main text. 


\section{Appendix D: Value of Optimum K for a Given Binding Energy}

The relationship of the optimum binding energy and kinetics (Eq. 23 of the main text) yields the following relationship between $K$ and $j$ :

$$
j=\frac{n F N k_{2}^{0} K\left(A^{4}-1\right)}{2 A \sqrt{K A^{2}+1} \sqrt{A^{2}+K}}
$$

By setting $k_{1}^{0} k_{2}^{0}=1$ as in the main text, the following expression can be obtained:

$$
j=\frac{n F N\left(A^{4}-1\right)}{2 A} \sqrt{\frac{K}{\left(K A^{2}+1\right)\left(A^{2}+K\right)}}
$$

Technically, this current density expression has a maximum at $K=1$, because the contents of the square root shows a minimum at $K=1$ based on:

$$
\frac{\left(K A^{2}+1\right)\left(K+A^{2}\right)}{K}=A^{4}+1+A^{2}\left(K+\frac{1}{K}\right)
$$

However, the plateau region is essentially flat and becomes larger upon increasing the driving force $(E)$ as shown in Figure 3 of the main text. Therefore, it should be possible to achieve activity similar to the optimum catalyst $\left(\log K=\Delta G_{H}=0\right)$, even with a catalyst with a non-zero $\Delta \mathrm{G}_{\mathrm{H}}$. 


\section{References in the Supporting Information}

(1) Pyun, C. W. Steady-state and equilibrium approximations in chemical kinetics. J. Chem. Educ. 48, 194 (1971). 